\title{
Analysis of Momordica charantia Polysaccharide Components and Their Effects on KA-Induced Oxidative Stress and Neuronal Loss in the Hippocampus of Epileptic Rats
}

\author{
Qiaoli Li1*, Nan Chen ${ }^{*}$, Heng Cai1, Yu Tang1, Xiaoyan Zhou1, Yue Huang1, Muxin Gong1, \\ Cunyu Qin', Xuewen Wei1,3, Suhua Qi1,2\# \\ ${ }^{1}$ Research Center for Biochemistry and Molecular Biology, Jiangsu Key Laboratory of Brain Disease Bioinformation, Xuzhou \\ Medical University, Xuzhou, China \\ ${ }^{2}$ School of Medical Technology, Xuzhou Medical University, Xuzhou, China \\ ${ }^{3}$ Department of Laboratory Medicine, Affiliated Municipal Hospital of Xuzhou Medical University, Xuzhou, China \\ Email: "suhuaqi@xzhmu.edu.cn
}

How to cite this paper: Li, Q.L., Chen, N., Cai, H., Tang, Y., Zhou, X.Y., Huang, Y., Gong, M.X., Qin, C.Y., Wei, X.W. and Qi, S.H. (2018) Analysis of Momordica charantia Polysaccharide Components and Their Effects on KA-Induced Oxidative Stress and Neuronal Loss in the Hippocampus of Epileptic Rats. World Journal of Neuroscience, 8, 113-123.

https://doi.org/10.4236/wjns.2018.82011

Received: December 21, 2017

Accepted: May 4, 2018

Published: May 7, 2018

Copyright (c) 2018 by authors and Scientific Research Publishing Inc. This work is licensed under the Creative Commons Attribution International License (CC BY 4.0).

http://creativecommons.org/licenses/by/4.0/

\section{c) (i) Open Access}

\begin{abstract}
Polysaccharides of Momordica charantia have been reported to be the primary bioactive components that confer its antioxidant activity. To some degree, antioxidants may provide beneficial effects on neuronal damage induced by seizures. However, the protective effects of Momordica charantia polysaccharides (MCPs) on seizures remain unclear. In this study, our purpose was to investigate the effects of MCPs on oxidative stress and neurodegeneration in an experimental Kainic acid (KA)-induced rat seizure model. MCPs treatments decreased the level of malondialdehyde (MDA) and increased the activities of superoxide dismutase (SOD) and catalase (CAT) in the hippocampus of the KA-induced animal model. Moreover, MCPs significantly attenuated KA-induced neuronal loss in the CA1 and CA3 hippocampal regions. Based on these results, MCPs exert neuroprotective effects by attenuating KA-induced neuronal damage in the brain through their free radical scavenging activities.
\end{abstract}

\section{Keywords}

KA-Induced Seizure, Oxidative Stress, Momordica charantia Polysaccharides, Antioxidant, Neuroprotective Effect

${ }^{\star}$ Contribute equally. 


\section{Introduction}

Epilepsy affects up to $1 \%$ of the general population and is characterized by recurrent, spontaneous and paroxysmal cerebral discharges with a miscellaneous etiology [1]. Based on accumulating evidence, antiepileptic drugs (AEDs) merely afford symptomatic relief from seizures, and some have no effects or exert intolerable adverse effects that lower the quality of life of up to $30 \%$ of patients with epilepsy. Moreover, these AEDs do not prevent the onset of epilepsy or block seizure progression [2]. Therefore, the design and discovery of new drugs and treatment strategies to ameliorate seizures and minimize side effects are required.

The underlying mechanisms of epilepsy remain largely unknown. Animal models of epilepsy and clinical studies have revealed that oxidative stress is one important mechanism in the pathogenesis of epilepsy, as it plays a significant role in the initiation and progression of epilepsy [3]. Oxidative stress is described as an imbalance between free radical generation and the antioxidant defense capacity in a biological system. Free radicals, including reactive oxygen species (ROS) and reactive nitrogen species (RNS), are products of cellular metabolism [4]. Excess generation of free radicals during epileptic seizures damages neuronal cells, inducing hippocampal neuronal cell loss through either a necrotic or an apoptotic pathway [5] [6]. Therefore, free radical scavenging agents may emerge as a beneficial strategy for treating epilepsy and protecting neurons. In fact, many antioxidants, such as lipoic acid, melatonin, resveratrol, $\alpha$-tocopherol and vitamin $\mathrm{C}$, have shown potential efficacy in treating convulsive disorders and neuroprotective effects on various seizure models [3].

Momordica charantia (MC) belongs to the Cucurbitaceae family and is also known as bitter melon. The fruit of MC is commonly consumed as a food but has also been used historically as a treatment for diabetes, cardiovascular diseases and cancer. M. charantia possesses antioxidant activity [7], anti-tumor activity [8], anti-obesity effects [9], hypoglycemic effects, the ability to reduce cholesterol levels [10] and other beneficial effects. Polysaccharides in $M$. charantia are the primary bioactive components that exert these different pharmacological effects of $M$. charantia. In addition to the aforementioned effects of $M$. charantia, studies from our laboratory have shown that Momordica charantia polysaccharides (MCPs) also have neuroprotective roles and potentially reduce thrombin-induced neuronal death in primary hippocampal neurons and decrease neuronal loss induced by cerebral ischemia/reperfusion (I/R) via inhibiting the oxidative stress-mediated JNK3 cellular signaling pathway [11] [12].

Kainic acid (KA), a nonselective and potent exogenous agonist of $\alpha$-amino-3-hydroxy-5-methyl-4-isoxazole propionate (AMPA) receptors and kainate receptors, is widely used to induce seizures. KA-induced animal models display similar clinical behaviors and pathological changes to patients with epilepsy. KA administration in rodents increases the generation of ROS and RNS, increases oxidative stress in brain tissues and induces neuron death via apoptosis and necrosis, specifically in the hippocampal CA1 and CA3 regions [13]. 
Based on the above findings, in the present study, we used a KA-induced Sprague-Dawley (SD) rat model to investigate the effects of MCPs on oxidative stress and neuronal cell loss in the hippocampus of epileptic rats. MCPs can protect neurons by their anti-oxidative effect, but there is no any research on epilepsy.

\section{Materials and Methods}

\subsection{Chemicals and Reagents}

In this study, KA was obtained from Sigma-Aldrich Corporation (Sigma-Aldrich, St. Louis, MO, USA). The SOD assay kit, CAT assay kit and MDA assay kit were purchased from Nanjing Institute of Biological Engineering (Nanjing, China). All other chemicals were obtained from Sigma Chemical Co., unless indicated otherwise.

\subsection{Preparation of MCPs and Chemical Identification}

Details of the procedures used to prepare MCPs were described in previous studies by Wu et al. [12] [14] and were performed with minor modifications. Briefly, MCPs were extracted from M. charantia with water by sonication, followed by the removal of starch and proteins and alcohol precipitation. MCP content was analyzed by high-performance liquid chromatography (HPLC) and gas chromatography (GC) of acetylated aldononitriles.

HPLC (Agilent1260, US, VWD detector) and experimental conditions are described below: ZORBAX Eclipse XDB-C ${ }_{18}(4.6 \times 250 \mathrm{~mm} 5 \mu \mathrm{m}$, Agilent1260); mobile phase: PBS (phosphate buffer solution)/acetonitrile (83:17) $8 \mathrm{mM}$; column temperature: $30^{\circ} \mathrm{C}$; flow rate: $1 \mathrm{~mL} / \mathrm{min}$; injection volume: $10 \mu \mathrm{L}$.

Ten milligrams of MCPs dissolved in distilled water $(1.6 \mathrm{~mL})$ were hydrolyzed with $1.6 \mathrm{~mL}$ of trifluoroacetic acid (TFA) $(4 \mathrm{~mol} / \mathrm{L})$ in a sealed glass tube with a screw cap at $110^{\circ} \mathrm{C}$ for $12 \mathrm{~h}$. The solution was evaporated, and $6 \mathrm{~mL}$ of methanol were added for further evaporation. This step was performed three times to completely remove the TFA.

The hydrolysate was treated with $40 \mathrm{mg}$ of hydroxylamine dissolved in $2 \mathrm{~mL}$ of a hydrochloride-pyridine solution and incubated in a sealed glass tube at $90^{\circ} \mathrm{C}$ for $30 \mathrm{~min}$. After cooling to room temperature, $2 \mathrm{~mL}$ of acetic anhydride were added and heated in the resealed tube at $90^{\circ} \mathrm{C}$ for another $30 \mathrm{~min}$. After cooling, the solution was evaporated to dryness. The dried residue was subjected to the analysis of the MCP components.

The gas chromatography (HP5890 SERIESI, US, FID detector) analysis was performed under the following conditions: DB-WAX capillary column $(30 \mathrm{~m} \times$ $320 \mu \mathrm{m}$ ); the initial temperature of the column was set to $180^{\circ} \mathrm{C}$, held for $3 \mathrm{~min}$, and then programmed to increase by $10^{\circ} \mathrm{C} / \mathrm{min}$ to $250^{\circ} \mathrm{C}$; a split injection $(1 \mu \mathrm{L})$ was applied with a split ratio of 1:50. High purity nitrogen was used as the carrier gas at a flow rate of $10 \mathrm{~mL} / \mathrm{min}$. The column temperature was $180^{\circ} \mathrm{C}$, and the sampling temperature was $250^{\circ} \mathrm{C}$. 


\subsection{Experimental Animals}

In our experiments, adult male SD rats $(270 \pm 30 \mathrm{~g}, 6$ - 8 weeks $)$ were provided by Shanghai Experimental Animal Center (Shanghai, China). All animal treatments and maintenance procedures were approved by the Animal Ethics Committee of Xuzhou Medical University (Approval ID: SCXK (SU) 2010-0003) and were performed according to the Guide for the Care and Use of Laboratory Animals from the National Institutes of Health. Rats were housed in standard cages in a controlled environment (constant temperature: $23^{\circ} \mathrm{C} \pm 2^{\circ} \mathrm{C}$; relative humidity: 50\% - 60\%; light period: 07:00-19:00) and were allowed free access to food and water ad libitum. After three days of acclimatization, rats were used for research purposes. We made all efforts to minimize the number of animals used, as well as their suffering.

Forty rats were randomly divided into four experimental groups to analyze the effects of MCPs on oxidative stress: a sham group (Sham), a control group (KA), a pre-KA group (Pre-KA) and a post-KA group (Po-KA). In the sham group, rats were intraperitoneally injected with $0.9 \%$ normal saline. In the control group, rats were intraperitoneally injected with $\mathrm{KA}(10 \mathrm{mg} / \mathrm{ml})$. In the pre-KA group, rats were intraperitoneally injected with MCPs $(50 \mathrm{mg} / \mathrm{kg}) 30$ min before the KA injection. In the post-KA group, MCPs $(50 \mathrm{mg} / \mathrm{kg})$ was i.e. administered to the rats $30 \mathrm{~min}$ after the KA injection [11] [12]. Both can have protective effects on the neurons, we gonna to investigate which is better of the preventive effect and the protective effect.

\subsection{Seizure Model}

Seizures were induced by the systemic injection of KA. KA $(10 \mathrm{mg} / \mathrm{ml})$ was dissolved in sterile normal saline and administered i.e. to the rats. After injection, the rats' behaviors were monitored to assess seizures, sluggish behavior, dull eyes, head scratching, unilateral forelimb myoclonus or bilateral forelimb myoclonus and even gradual loss of positional sensation after the occurrence of several seizures. Five to six rats from each group were selected $(n=10$, model success rate of approximately $50 \%-60 \%$ ).

\subsection{Sample Preparation}

Six hours after KA injection, rats were treated with ether anesthetic and were immediately decapitated using a guillotine. The hippocampus was quickly and carefully dissected from both hemispheres and rapidly frozen in liquid nitrogen. The hippocampus was thawed and homogenized in 10\% (w/v) ice-cold homogenization buffer (pH 7.4) containing $320 \mathrm{mM}$ sucrose, $100 \mathrm{mM} \mathrm{KCl}, 50 \mathrm{mM} \mathrm{NaF}$, $50 \mathrm{mM}$ 3-(N-morpholino) propanesulfonic acid (MOPS), $20 \mathrm{mM}$ $\beta$-phosphoglycerol, $20 \mathrm{mM}$ sodium pyrophosphate, $1 \mathrm{mM}$ p-nitrophenyl phosphate (PNPP), $1 \mathrm{mM}$ EDTA, $1 \mathrm{mM}$ EGTA, $1 \mathrm{mM}$ phenylmethylsulfonyl fluoride (PMSF), $1 \mathrm{mM}$ benzamidine, $1 \mathrm{mM} \mathrm{Na}_{3} \mathrm{VO}_{4}, 0.5 \mathrm{mM} \mathrm{MgCl}, 0.2 \mathrm{mM}$ DTT and 5 $\mathrm{mg} / \mathrm{ml}$ each of aprotinin, leupeptin and pepstatin A. Homogenates were centri- 
fuged at $800 \mathrm{~g}$ for $10 \mathrm{~min}$ at $4^{\circ} \mathrm{C}$. The resulting supernatants were collected, and the protein content was determined using the Lowry method [15]. Samples were stored in aliquots at $-80^{\circ} \mathrm{C}$ and thawed once before use.

\subsection{Lipid Peroxidation and Antioxidant Enzyme Assays}

To study the antioxidant capacity of MCPs, we measured SOD and CAT activities and the MDA levels in hippocampus samples using purchased kits Liu [16]. The assays were conducted according to the manufacturer's instructions.

\subsection{Morphological Examination}

Seven days after KA injection, rats were deeply anesthetized with chloral hydrate $(350 \mathrm{mg} / \mathrm{kg}$, i.e.) and transcardially perfused with ice-cold normal saline followed by $4 \%$ paraformaldehyde in $0.1 \mathrm{M}$ sodium phosphate buffer ( $\mathrm{pH}$ 7.4). Brains were removed quickly and further fixed with the same fixation solution overnight at $4^{\circ} \mathrm{C}$. Post-fixed brains were embedded in paraffin, and coronal sections $(5-\mu \mathrm{m}$ thick) were prepared using a microtome. The paraffin-embedded brain sections were deparaffinized with xylene and rehydrated with graded concentrations of $100-70 \%(\mathrm{v} / \mathrm{v})$ ethanol, followed by washes with water. Sections were stained with $0.1 \%(\mathrm{w} / \mathrm{v})$ cresyl violet and examined under a light microscope. The number of surviving hippocampal CA1 and CA3 pyramidal cells/1 $\mathrm{mm}$ of length was counted to establish the neuronal density. Cells counted under an Olympus BH-2 microscope at 400x magnification. The optical dissector technique was employed to avoid double counting cells.

\subsection{Statistical Analysis}

The experimental data were processed using SPSS 22 statistical analysis software. All data are expressed as means \pm SEM. Statistical analyses of the results were performed using Student's t-test or one-way analysis of variance, followed by Duncan's new multiple range method or the Newman-Keuls test. P-values $<0.05$ were considered significant.

\section{Results}

\subsection{Analysis of Momordica charantia Polysaccharide Components}

HPLC and GC analyses were used to identify the components of the extracted MCPs. Based on the HPLC data, MCPs contain galacturonic acid, glucose, and galactose (Figure 1), and the GC analysis showed that the MCPs contained glucose, galactose, rhamnose and fructose (Figure 2). Thus, MCPs contain galacturonic acid, glucose, galactose, rhamnose and fructose.

\subsection{MCPs Inhibit Lipid Peroxidation and Increases Antioxidant Enzyme Activities in the Hippocampi of Seizure Models}

MDA is an indicator of lipid peroxidation and is a marker of brain oxidative stress. SOD and CAT are antioxidant biomarkers in the tissue samples. We 


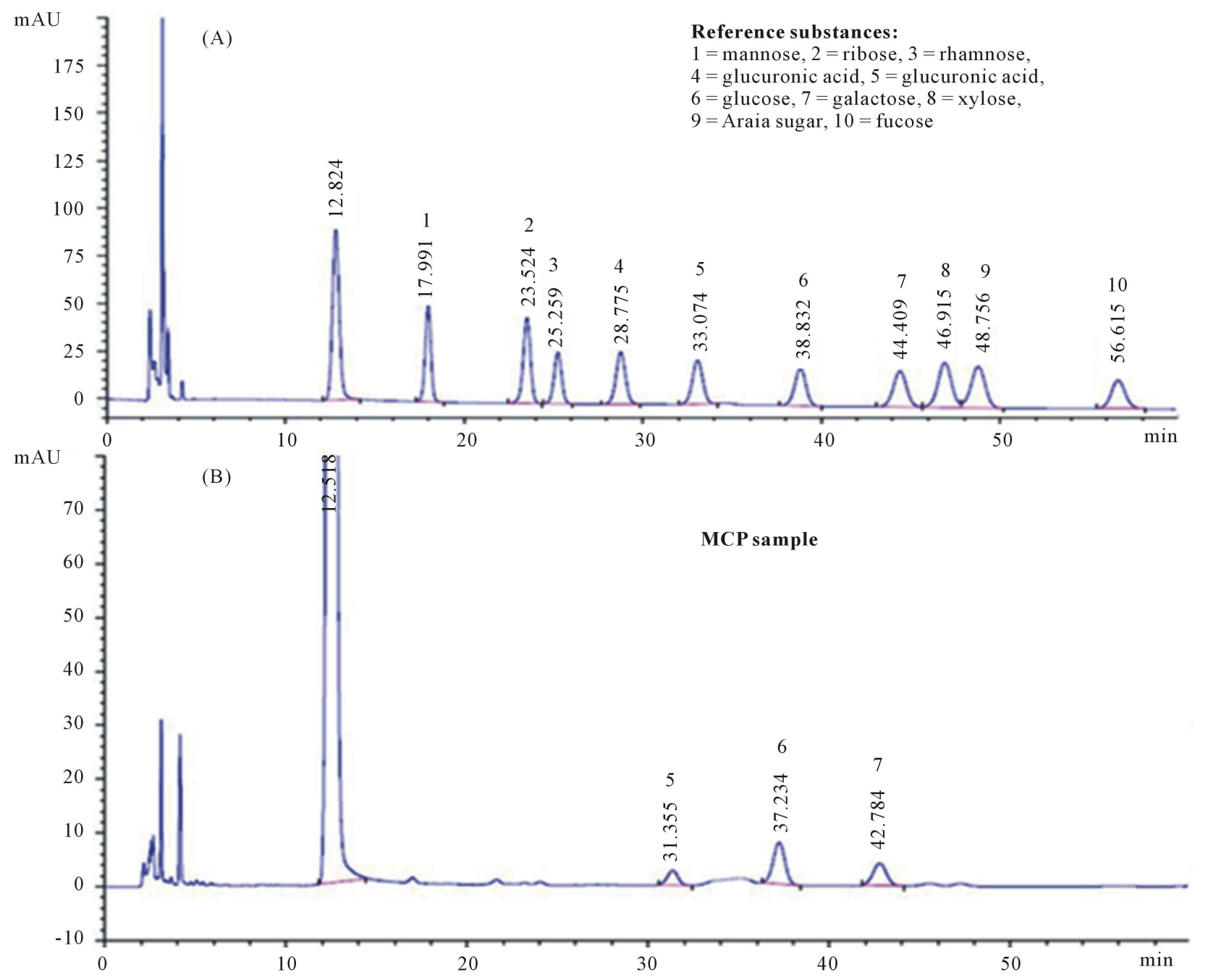

Figure 1. High-performance liquid chromatography analysis of the MCP content (A) HPLC chromatogram of reference substances. The chromatogram of reference substances and the MCP sample were acquired on a ZORBAX Eclipse XDB-C18 (4.6 $\times$ $250 \mathrm{~mm} 5 \mu \mathrm{m}$, Agilent1260) with the mobile phases: BS (phosphate buffer solution)/acetonitrile (83:17) $8 \mathrm{mM}$. The retention times for the reference standards and every substance, including ten monosaccharide compositions, were: 1-17.991, 2-23.524, 3-25.259, 4-28.775, 5-33.074, 6-38.832, 7-44.409, 8-46.916, 9-48.786, and 10-56.616 for mannose, ribose, rhamnose, galacturonic acid, galacturonic acid, glucose, galactose, xylose, arabinose and fucose, respectively; a, standard reference; (b) The ratio of every monosaccharide component in MCPs. Samples were detected using HPLC. The ratio of the content of every monosaccharide component was determined. Areas of peaks 5-7 corresponded to three different monosaccharides, galacturonic acid, glucose, and galactose, at a molar ratio of 1:4:2.

measured the MDA accumulation and SOD and CAT activities in hippocampal tissue from KA-induced seizure model to elucidate the mechanism underlying the antioxidant effects of MCPs. As shown in Table 1, the administration of 50 $\mathrm{mg} / \mathrm{kg}$ MCP before or after KA injection significantly decreased the MDA levels and remarkably increased the activities of SOD and CAT enzymes.

\subsection{MCPs Attenuate KA-Induced Neuron Loss in the Hippocampal CA1 and CA3 Regions}

Cresyl violet staining was used to examine the survival of pyramidal cells in the 


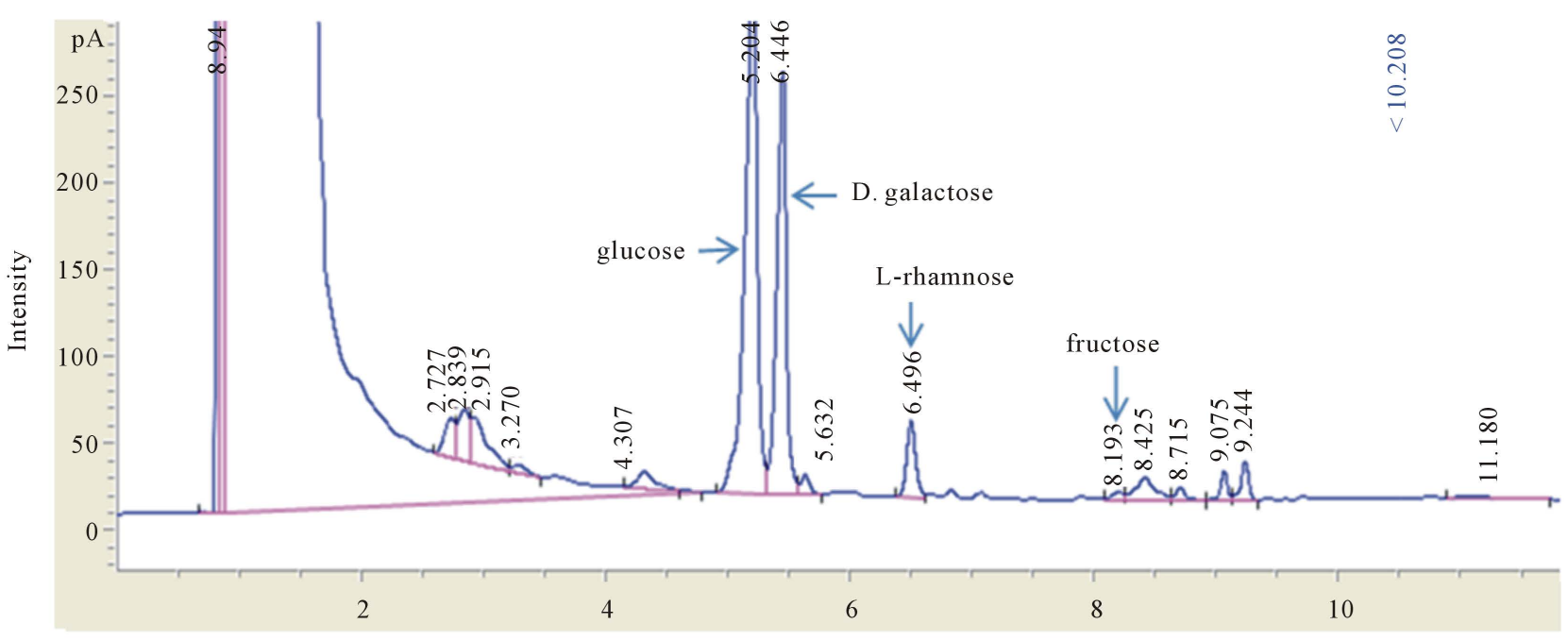

Time (min)

Figure 2. Gas chromatography analysis (A) The chromatogram of MCPs was achieved using gas chromatography (HP5890 SERIESI, US, FID detector). The analysis was performed under the following conditions: DB-WAX capillary column $(30 \mathrm{~m} \times 320$ $\mu \mathrm{m})$ with the mobile phases: high purity nitrogen at a flow rate of $10 \mathrm{~mL} / \mathrm{min}$. The retention times of the reference standard and every substance, including seven monosaccharide components (fructose, galactose, xylose, mannose, glucose, rhamnose and arabinose) are shown; a, standard reference. Seven samples were detected using GC. Areas of peaks 9, 10, 12, and 13 corresponded to four different monosaccharides: glucose, galactose, rhamnose and fructose.

Table 1. Effects of MCPs on SOD and CAT activities and MDA concentrations in brain tissues from rats with KA-induced seizures.

\begin{tabular}{cccc}
\hline & SOD (U/mg protein) & CAT (U/mg protein) & MDA (nmol/mg protein) \\
\hline Sham & $101.2 \pm 11.37$ & $0.21 \pm 0.03$ & $0.39 \pm 0.06$ \\
KA & $109.5 \pm 10.36$ & $0.38 \pm 0.04 \#$ & $0.41 \pm 0.05^{*}$ \\
Pre-50 & $169.3 \pm 15.97^{*}$ & $0.67 \pm 0.06^{*}$ & $0.27 \pm 0.03^{*}$ \\
Po-50 & $137.5 \pm 12.88^{*}$ & $0.53 \pm 0.05^{*}$ & $0.32 \pm 0.04^{*}$ \\
\hline
\end{tabular}

Notes: MCPs were administered at a dose of $50 \mathrm{mg} / \mathrm{kg}$ prior to (Pre-50) and after the KA injection (Po-50). Data are expressed as means \pm SEM ( $\mathrm{n}=6$ ), \#p < 0.05 compared with the sham group, ${ }^{*} \mathrm{p}<0.05$ compared with the KA group.

CA1 and CA3 regions of the hippocampus and the neuroprotective effects of MCPs on KA-induced neuronal damage. As shown in Figure 3, the administration of the MCP $(50 \mathrm{mg} / \mathrm{kg})$ treatment before or after KA injection remarkably suppressed KA-induced neuron loss in the hippocampal CA1 and CA3 regions.

\section{Discussion}

In the present study, MCPs attenuated the KA-induced MDA levels and increased the SOD and CAT activities in the hippocampus (Table 1). Moreover, MCPs significantly reduced KA-induced neuronal loss in the CA1 and CA3 regions of the hippocampus (Figure 3). Thus, MCPs exert a neuroprotective effect due to their ability to attenuate KA-induced neuronal damage in the brain, which is likely due to their ability to scavenge free radicals. 
(A)

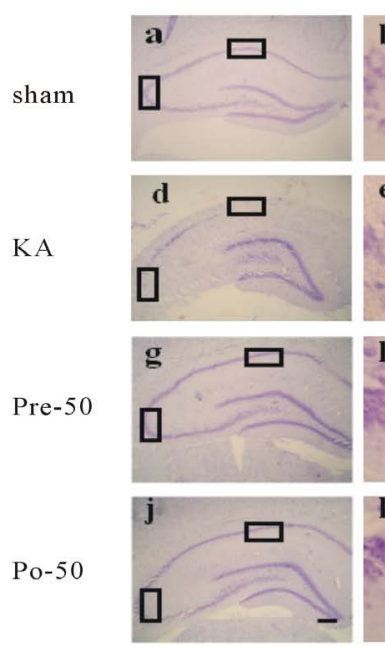

CA 1
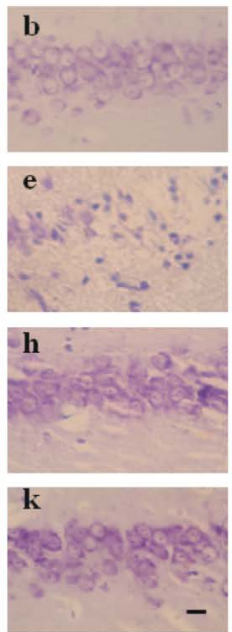

$\mathrm{CA} 3$
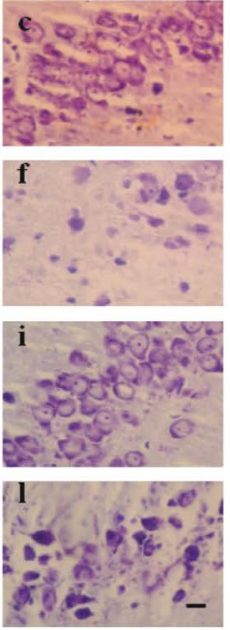

(B)

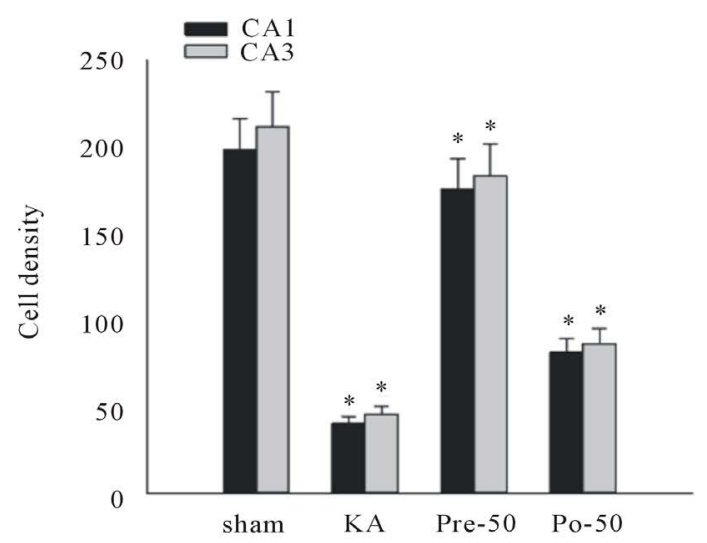

Figure 3. Neuroprotective effect of MCP on KA-induced neuronal injury (A) The survival of hippocampal pyramidal cells was analyzed using cresyl violet staining. Rats were injected with sterile normal saline ( $a, b$ and $c$ ) or KA (d, e and f) for 7 days and were pre-treated with MCPs at a dose of $50 \mathrm{mg} / \mathrm{kg}$ (g, h and i) $30 \mathrm{~min}$ before the KA injection. MCPs were also administered to the rats at a dose of $50 \mathrm{mg} / \mathrm{kg}$ (j, $\mathrm{k}$ and l) $30 \mathrm{~min}$ after the KA injection. The magnification in panels a, $\mathrm{d}, \mathrm{g}$ and $\mathrm{j}$ is $40 \times$; scale bars represent $200 \mu \mathrm{m}$. The magnification in panels b, c, e, f, h, i, k and lis $400 \times$; scale bars represent $20 \mu \mathrm{m}$. (B) Cell density is expressed as the number of cells per $1 \mathrm{~mm}$ length of the CA1 and CA3 pyramidal cells counted under a light microscope. ${ }^{*} \mathrm{p}<0.05$ compared with the saline group; $\# \mathrm{p}<0.05$ compared with the KA-injected groups $(\mathrm{n}=5)$.

Compared with other organs, the brain consumes the greatest amount of oxygen and produces high quantities of free radicals, and thus the brain is particularly susceptible to oxidative stress. Free radicals are both essential and detrimental to life. Normally, ROS destroy exogenous microorganisms and if produced in excess quantities, they even induce cell damage and apoptosis [17] [18]. The balance between the generation and elimination of free radicals is certainly crucial to maintain the normal function of cerebral neurons. According to many experimental reports, oxidative stress produced by excess free radicals plays an important role in the initiation and progression of epilepsy, and the changes attributed to oxidative stress are the main mechanism by which neuronal cells undergo death [19] [20]. For this reason, the brain must maintain oxidative balance and control, and a beneficial strategy for epilepsy would be to target oxidative stress components [3]. We extracted MCPs from M. charantia and analyzed the components using HPLC and gas chromatography. Galacturonic acid was the main component of MCPs; the other components were glucose, galactose, xylose, rhamnose and mannose (Figure 1 and Figure 2). As shown in our previous studies, MCPs protect against cerebral I/R injury via their potential to mitigate the oxidative stress burden [12]. Therefore, we investigated the antioxidant effects of MCPs on KA-induced epileptic rats in the present study.

In subjects with various epileptic conditions, the formation of free radicals that cause lipid peroxidation, an unavoidable outcome of ROS injury in the cellular and subcellular membranes, increases. MDA is a direct product of lipid peroxidation, which is the most commonly used indicator of the severity of lipid 
peroxidation and a marker of oxidative stress in the brain. Under normal physiological conditions, the generation of free radicals is controlled by the antioxidant defense system, which includes enzymatic and non-enzymatic mechanisms. Glutathione and uric acid are non-enzymatic antioxidants, whereas the most important enzymatic antioxidants are superoxide dismutase, catalase, and glutathione peroxidase (GPX) [21]. The roles of CAT and SOD in protecting against free radical damage have been widely studied and are well known. Thus, in the present study, we investigated the antioxidant properties of MCPs in inhibiting KA-induced neuronal damage by determining the levels of MDA and the activities of SOD and CAT in the hippocampus. Based on our results, pre- or post-treatment with MCPs remarkably decreased the MDA levels and increased the SOD and CAT activities in the hippocampal homogenates from KA-treated rats (Table 1). This finding validated the antioxidant properties of MCPs in the KA-induced seizure model. Oxidative stress produced by free radicals is an important cause and consequence of seizures [22]. Excess free radicals can induce tissue injury and activate apoptosis [17], resulting in neuron loss. As shown in Figure 3, morphological analyses performed using cresyl violet staining confirmed that pre- or post-treatment with MCPs generated neuroprotective effects on epileptic brain damage, as observed in the KA-induced rat seizure model. As shown in previous studies from our laboratory, MCPs exert neuroprotective effects on neuronal damage induced by cerebral I/R via their antioxidant properties of scavenging free radicals. This scavenging activity subsequently inhibits the apoptotic signaling pathway, including both the extrinsic and intrinsic pathways [12]. Based on the results from our current and previous studies, we conclude that pre- or post-treatment with MCPs directly scavenges free radicals, which at least partially contributes to their neuroprotective effects on KA-induced neuronal injury. These findings provide a direction and a promising approach to the prophylaxis and treatment of epilepsy. Of course, further studies are needed to elucidate the complete neuroprotective mechanism of MCPs and their therapeutic potential.

Our research has analyzed the components of MCPs, finding the anti-oxidative effect of MCPs on epilepsy, which can be used to the prevention and protection of epilepsy.

\section{Acknowledgements}

This work was supported by grants from the National Natural Science Foundation of China (Grant No. 81671164) and the Natural Science Foundation of Jiangsu Six big Talent Peak, and a project was funded by the Priority Academic Program Development of Jiangsu Higher Education Student Research Train Program (SRTP) (No. 201510313014Z).

\section{References}

[1] Fiest, K.M., Sauro, K.M., Wiebe, S., Patten, S.B., Kwon, C.S., Dykeman, J., Pring- 
sheim, T., Lorenzetti, D.L. and Jetté, N. (2017) Prevalence and Incidence of Epilepsy: A Systematic Review and Meta-Analysis of International Studies. Neurology, 88, 296-303. Https://doi.org/10.1212/WNL.0000000000003509

[2] Kwan, P., Arzimanoglou, A., Berg, A.T., Brodie, M.J., Allen Hauser, W., Mathern, G., Moshe, S.L., Perucca, E., Wiebe, S. and French, J. (2010) Definition of Drug Resistant Epilepsy: Consensus Proposal by the Ad Hoc Task Force of the ILAE Commission on Therapeutic Strategies. Epilepsia, 51, 1069-1077.

https://doi.org/10.1111/j.1528-1167.2009.02397.x

[3] Azam, F., Prasad, M.V. and Thangavel, N. (2012) Targeting Oxidative Stress Component in the Therapeutics of Epilepsy. Current Topics in Medicinal Chemistry, 12, 994-1007. https://doi.org/10.2174/156802612800229224

[4] Dar, I. and Barzilai, A. (2009) Cellular Responses to Oxidative Stress. In: Khanna, K.K. and Shiloh, Y., Eds., The DNA Damage Response Implications on Cancer Formation and Treatment, Springer, Dordrecht, 65-79. https://doi.org/10.1007/978-90-481-2561-6_4

[5] Mikati, M.A., Abi-Habib, R.J., El Sabban, M.E., Dbaibo, G.S., Kurdi, R.M., Kobeissi, M., Farhat, F. and Asaad, W. (2003) Hippocampal Programmed Cell Death after Status Epilepticus: Evidence for NMDA-Receptor and Ceramide-Mediated Mechanisms. Epilepsia, 44, 282-291. https://doi.org/10.1046/j.1528-1157.2003.22502.x

[6] Shin, E.J., Jeong, J.H., Chung, Y.H., Kim, W.K., Ko, K.H., Bach, J.H., Hong, J.S., Yoneda, Y. and Kim, H.C. (2011) Role of Oxidative Stress in Epileptic Seizures. Neurochemistry International, 59, 122-137. https://doi.org/10.1016/j.neuint.2011.03.025

[7] Shan, B., Xie, J.H., Zhu, J.H. and Peng, Y. (2012) Ethanol Modified Supercritical Carbon Dioxide Extraction of Flavonoids from Momordica charantia L. and Its Antioxidant Activity. Food \& Bioproducts Processing, 90, 579-587.

https://doi.org/10.1016/j.fbp.2011.09.004

[8] Nerurkar, P. and Ray, R.B. (2010) Bitter Melon: Antagonist to Cancer. Pharmaceutical Research, 27, 1049-1053. https://doi.org/10.1007/s11095-010-0057-2

[9] Chan, L.L., Chen, Q., Go, A.G., Lam, E.K. and Li, E.T. (2005) Reduced Adiposity in Bitter Melon (Momordica charantia) - Fed Rats Is Associated with Increased Lipid Oxidative Enzyme Activities and Uncoupling Protein Expression. The Journal of Nutrition, 135, 2517-2523. https://doi.org/10.1093/jn/135.11.2517

[10] Jayasooriya, A.P., Sakono, M., Yukizaki, C., Kawano, M., Yamamoto, K. and Fukuda, N. (2000) Effects of Momordica charantia Powder on Serum Glucose Levels and Various Lipid Parameters in Rats Fed with Cholesterol-Free and Cholesterol-Enriched Diets. Journal of Ethnopharmacology, 72, 331-336. https://doi.org/10.1016/S0378-8741(00)00259-2

[11] Duan, Z.Z., Zhou, X.L., Li, Y.H., Zhang, F., Li, F.Y. and Qi, S.H. (2015) Protection of Momordica charantia Polysaccharide against Intracerebral Hemorrhage-Induced Brain Injury through JNK3 Signaling Pathway. Journal of Receptors and Signal Transduction, 35, 523-529. https://doi.org/10.3109/10799893.2014.963871

[12] Gong, J., Sun, F., Li, Y., Zhou, X., Duan, Z., Duan, F, Zhao, L., Chen, H., Qi, S. and Shen, J. (2015) Momordica charantia Polysaccharides Could Protect against Cerebral Ischemia/Reperfusion Injury through Inhibiting Oxidative Stress Mediated c-Jun N-terminal Kinase 3 Signaling Pathway. Neuropharmacology, 91, 123-134. https://doi.org/10.1016/j.neuropharm.2014.11.020

[13] Simonian, N.A., Getz, R.L., Leveque, J.C., Konradi, C. and Coyle, J.T. (1996) Kainic Acid Induces Apoptosis in Neurons. Neuroscience, 75, 1047-1055. 
https://doi.org/10.1016/0306-4522(96)00326-0

[14] Wu, X., Wang, Z., Cai, Y., Yang, Y. and Wang, M. (2011) Extraction, Isolation, Purification and Composition of Momordica charantia Polysaccharide. Chinese Journal of Bioprocess Engineering, 9, 19-23.

[15] Lowry, O.H., Rosebrough, N.J., Farr, A.L. and Randall, R.J. (1951) Protein Measurement with the Folin Phenol Reagent. Journal of Biological Chemistry, 193, 265-275.

[16] Liu, X., Chen, T., Hu, Y., Li, K. and Yan, L. (2014) Catalytic Synthesis and Antioxidant Activity of Sulfated Polysaccharide from Momordica charantia, L. Biopolymers, 101, 210-215. https://doi.org/10.1002/bip.22312

[17] Todorova, V.K., Harms, S.A., Kaufmann, Y., Luo, S., Luo, K.Q., Babb, K. and Klimberg, V.S. (2004) Effect of Dietary Glutamine on Tumor Glutathione Levels and Apoptosis-Related Proteins in DMBA-Induced Breast Cancer of Rats. Breast Cancer Research and Treatment, 88, 247-256. https://doi.org/10.1007/s10549-004-0783-4

[18] Zhang, H., Slutsky, A.S. and Vincent, J.L. (2000) Oxygen Free Radicals in ARDS, Septic Shock and Organ Dysfunction. Intensive Care Medicine, 26, 474-476. https://doi.org/10.1007/s001340051185

[19] Akdogan, I. and Yonguc, N.G. (2011) Experimental Epilepsy Models and Morphologic Alterations of Experimental Epilepsy Models in Brain and Hippocampus. In: Kaneez, F.S., ed., Underlying Mechanisms of Epilepsy, InTech, Croatia, 269-282. https://doi.org/10.5772/19928

[20] Andres-Mach, M., Fike, J.R. and Luszczki, J.J. (2011) Neurogenesis in the Epileptic Brain: A Brief Overview from Temporal Lobe Epilepsy. Pharmacological Reports, 63, 1316-1323. https://doi.org/10.1016/S1734-1140(11)70696-X

[21] Ilhan, A., Aladag, M.A., Kocer, A., Boluk, A., Gurel, A. and Armutcu, F. (2005) Erdosteine Ameliorates PTZ-Induced Oxidative Stress in Mice Seizure Model. Brain Research Bulletin, 65, 495-499. https://doi.org/10.1016/j.brainresbull.2005.02.027

[22] Aguiar, C.C., Almeida, A.B., Araujo, P.V., de Abreu, R.N., Chaves, E.M., do Vale, O.C., Macedo, D.S., Woods, D.J., Fonteles, M.M. and Vasconcelos, S.M. (2012) Oxidative Stress and Epilepsy: Literature Review. Oxidative Medicine \& Cellular Longevity, 2012, Article ID: 795259. https://doi.org/10.1155/2012/795259 